\title{
Acute renal failure secondary to lupus nephritis confirmed by renal biopsy with an unusually normal immunological profile
}

\author{
Zhigang Liu ${ }^{1 *}$, Andrew Ofosu ${ }^{1}$, Richard Williams ${ }^{1}$, Navdeep Grewal ${ }^{2}$ \\ ${ }^{1}$ Department of Medicine, MedStar Harbor Hospital, Baltimore, USA; *Corresponding Author: zgliu0805@yahoo.com \\ ${ }^{2}$ Ross University School of Medicine, Roseau, Commonwealth of Dominica
}

Received 5 December 2013; revised 30 December 2013; accepted 10 January 2014

Copyright (C) 2014 Zhigang Liu et al. This is an open access article distributed under the Creative Commons Attribution License, which permits unrestricted use, distribution, and reproduction in any medium, provided the original work is properly cited. In accordance of the Creative Commons Attribution License all Copyrights (C) 2014 are reserved for SCIRP and the owner of the intellectual property Zhigang Liu et al. All Copyright (C) 2014 are guarded by law and by SCIRP as a guardian.

\section{ABSTRACT}

The gold standard to diagnose the lupus nephritis is the renal biopsy. It provides information not only for diagnosis but also for the treatment plan and the prognosis. Laboratory studies, including the immunological profile, play an essential role in diagnosing and evaluating the lupus nephritis activity. The patient is unlikely to have the active lupus nephritis with the combination of anti-ds DNA, anti-C1q, C3 and C4 being within normal limits. In this case report, we present a patient with moderately active diffuse proliferative lupus glomerulonephritis (Class IV) confirmed by the renal biopsy, while her immunological profile is unusually normal.

\section{KEYWORDS}

\section{Lupus Nephritis; Anti-Ds DNA; C3, C4;} Immunological Profile

\section{INTRODUCTION}

This Lupus nephritis (LN) is one of the most common manifestations of systemic lupus erythematous (SLE), with the prevalence varying from $29 \%$ to $65 \%$ during the lifetime of lupus patients [1]. Renal involvement in SLE carries significant morbidity and mortality. The spectrum of LN is wide, from asymptomatic proteinuria or hematuria to overt nephritic and nephrotic syndromes. End stage renal failure is found in up to $25 \%$ of patients 10 years after the onset of renal disease [2]. As a result, it is critical to recognize and treat LN promptly, as better prognosis is correlated to the early response to therapy [3].

The gold standard to confirm the diagnosis and flare of
LN is renal biopsy. A renal biopsy, with optical microscope, immunofluorescence staining and electron microscope, is the key to the ISN/RPS classification [4]. In addition, it also provides essential information for the prognosis and treatment plan. However, renal biopsy is an invasive procedure, carrying its own risks. There are strict indications for renal biopsy or even repeating renal biopsies [5].

Other laboratory studies, including proteinuria, createnine, anti-ds DNA, complement level (C3, C4) and anti-C1q, are helpful in the diagnosing and monitoring LN activity. Anti-C1q is very specific for the renal activity, but it is not widely available yet [6]. Some patients also have circulating anti-neutrophil cytoplasmic antibodies, but no specific clinical significance is found. The specificity of the immunological marker combination is high for LN. The patient is very unlikely to have active LN if anti-ds DNA, C3, C4 and anti-C1q are within normal limits $[7,8]$.

\section{CASE REPORT}

A 48-year-old Hispanic female presented to emergency room with two weeks of constant frank hematuria.

Past medical history was significant for SLE diagnosed in 2000 with joint pain, hair loss and photosensitive rash. In addition, patient had fibromyalgia, mitral valve prolapse, gastroesophageal reflux disease, increased anticardiolipin titers, migraine, and chronic kidney disease stage 3 . The patient family history included mother having rheumatoid arthritis, hypertension, and father having hypertension, hyperlipidemia, gout, and diabetes mellitus.

The patient experienced constant frank hematuria in the last two weeks, which she never had before. In addi- 
tion, she also complained of poor appetite, nausea, nonbloody vomiting. The exercise tolerance was decreased with progressive exertional dyspnea and bilateral pedal edema.

Patient's home medication included hydroxychloroquine $200 \mathrm{mg}$ bid, gabapentin $300 \mathrm{mg}$ PO tid, Oxycodone 15 mg PO q4h p.r.n, ranitidine 150 mg PO bid. Patient had been taking hydroxychloroquine, prescribed by her rheumatologist, for several years without major side effects, and actively following up with her rheumatologist. No other renal toxic medication was in her medication list. No new medication was added recently. Patient also denied smoking, alcohol abuse and illicit drug abuse.

Physical examination on admission: well-nourished, obviously nauseous. Vital signs: temperature $\mathbb{Z}, 2$ pulse 89 per minute, blood pressure 169/79 mmHg, respiration rate 22 per minute, oxygen saturation $99 \%$ on room air. HEENT: normocephalic and atraumatic, pale conjunctivae, dry mucosa. Neck: supple, no bruits, jugular venous distention 3 to $4 \mathrm{~cm}$. Lungs: a few coarse rales bibasilarly. Heart: regular rhythm, normal S1 and S2, no murmurs, rub or gallop. Abdomen: soft, nontender, nondistended, normal bowel sounds, no costovertebral angle tenderness. Extremities: mild edema of the lower extremities. Neurology: awake, alert, oriented $\times 3$, no focal neurological deficit.

Chest $\mathrm{X}$ ray showed mild bibasilar pulmonary edema. Renal ultrasound demonstrated that both kidneys were $10.3 \mathrm{~cm}$, no evidence of renal mass, hydronephrosis or nephrolithiasis. EKG was normal with sinus rhythm.

Laboratory results on this admission: sodium 142, potassium 3.9, glucose random 94, BUN 58, Creatinine 10.6, calcium 7.4, magnesium 1.3, WBC 5.3, Hgb 6.0, Hct 17.7, platelet 270, PT 13.9, INR 1.1, PTT 39.7, reticulocyte count 1.0\%, LDH 217, haptoglobin 281, TIBC 200, Iron Saturation 27\%, iron 54, ferritin 84.8, ANA screen negative, p-ANCA positive, c-ANCA negative, anti-ds DNA antibody negative, glomerular BM antibody negative, borderline low normal C3 92 and C4 16.4, 24 hour urine protein $5104 \mathrm{mg}$, urine analysis: RBC 564/ HPF, 3 hyaline cast, protein $414 \mathrm{mg} / \mathrm{dl}$. Previous laboratory results: Creatinine 1.95, BUN 27 one month ago, Hgb 10.2 and Hct 29.6 two months ago.

Patient was admitted for acute renal failure with possible etiology of lupus nephritis. The acute anemia was most likely due to persistent hematuria, and patient was transfused with pack red blood cell. Urgent hemodialysis was started because of her rapidly deteriorating renal function. Empirical high dose of methylprednisolone and cyclophosphamide were administrated intravenously despite the normal immunological profile, including C3, C4, antids DNA antibody and ANA.

To ascertain the etiology of the acute kidney failure, kidney biopsy was performed. The samples were sent for optical microscope, immunofluorescence techniques, and an electron microscope analysis. The pathology slides were shown as in Figures 1 and 2. Light microscopy results: diffuse necrotizing and crescentic proliferative glomerulonephritis, cellular crescents in at least 25\% glomeruli, mild segmental thickening of the capillary loop, fibrin and fibrinoid necrosis in 5\% glomeruli, moderate mononuclear interstitial inflammation and mild edema with occasional plasma cells , mild to moderate intestinal fibrosis in $25 \%$ cortex, moderate to severe acute tubular injury. Immunofluorescence microscopy results: C1q trace to $1+, \mathrm{C} 32+$, IgA trace, IgG 3+, LgM $2+$, kappa 3+, lambda 2+, albumin 2+. Electron microscopy results: variable thickening of the glomeruli basement membranes, extensive ischemic wrinkling of the capillary wall, scattered intramembranous electron dense deposits in the capillary wall, severe podocyte injury.

The pathology results confirmed the diagnosis of diffuse proliferative lupus glomerulonephritis (Class IV) with moderate activity.

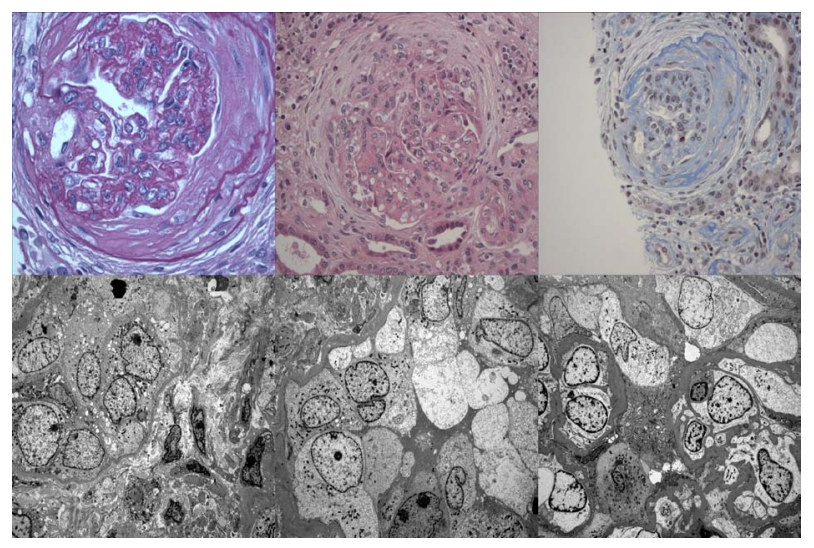

Figure 1. Optical microscope, and electron microscope studies. Upper panel: optical microscope (original magnification $\times 400$ ), Lower panel: electron microscope (original magnification $x$ 8000).

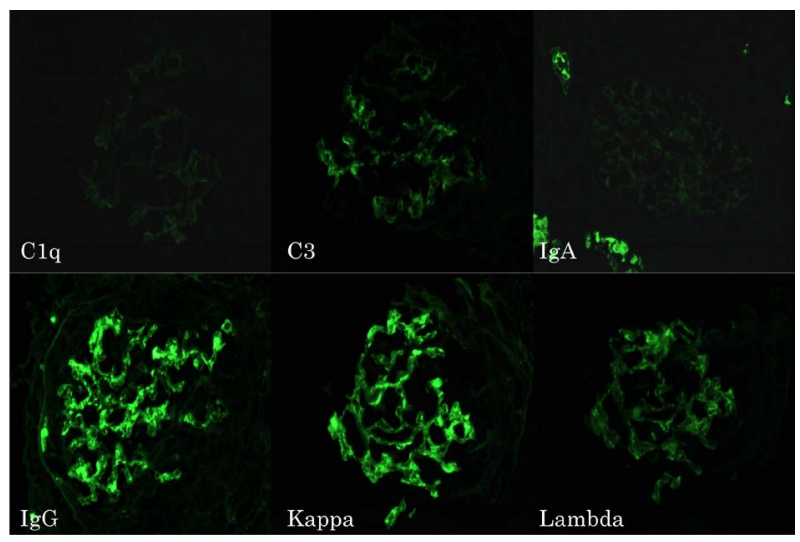

Figure 2. Immunofluorescence staining, the different staining is labeled in each panel (original magnification $\times 400$ ). 
Despite the prompt diagnosis and appropriate treatment with methylprednison and cyclophosphamide induction followed by low dose of steroids, the kidney function failed to recover during one year long follow up, and chronic hemodialysis was required for renal replacement therapy.

\section{DISCUSSION}

In this case, we report a 48-year-old Hispanic female with acute kidney failure due to the moderately active diffuse proliferative lupus glomerulonephritis confirmed by renal biopsy, however, her immunological profile, including $\mathrm{C} 3, \mathrm{C} 4$, anti-ds DNA and ANA, was within the normal range. Although anti-C1q was not measured, it was reasonable to assume that the level was not significantly elevated, considering the immunofluorescence staining of $\mathrm{C} 1 \mathrm{q}$ was trace to $1+$ only on the glomeruli. Given the good negative predictive value of the combination of these immunological markers, the normal values are actually abnormal for the patient with active LN [7].

Proteinuria is reported in $100 \%$ of patients with LN, with $45 \%$ to $65 \%$ patients having nephrotic syndrome. However, the severity of proteinuria is not closely related to the activity of LN. According to a respective study, as many as 12 out of 21 patients diagnosed with proliferative lupus nephritis by renal biopsy were found to have proteinuria less than $1 \mathrm{~g}$ /day [9]. Microscopic hematuria is discovered in $80 \%$ of $\mathrm{LN}$ case, although macroscopic hematuria is rare. About one-half of all patients have a reduced glomerular filtration rate, with occasional initial presentation of acute kidney injury [10].

Ethnicity plays an important role in the severity and prognosis of LN. It is found that patients with Hispanic and Asian original suffer more from the frequency and severity of LN, compared to Caucasians. In addition, end stage renal disease is more common in these patient groups [11]. The patient in this case report, a Hispanic female who is presented with acute kidney failure, required urgent hemodialysis. Moreover, she did not respond well to treatment and she required the long-term hemodialysis.

The class IV diffuse LN is the most frequently biopsied form. This form of LN usually presents with hematuria, proteinuria, nephrotic syndrome, renal failure and hypertension. Moreover, compared to other classes of LN, class IV is more likely to be associated with elevated anti-ds DNA and hypocomplementaemia [12]. The patient in this case reported was diagnosed with class IV LN with all typical clinical presentations. However, her immunological profile is unusually within normal range.

Given the poor prognosis of LN without early detection and therapy, it is urgent to identify biomarkers that are sensitive and specific to the disease activity and therapy response. Extensive investigation has been performed to detect the different gene expression pattern during active and inactive LN [13]. Several promising biomarkers have been identified, such as monocyte chemoattractant protein-1 (MCP-1), neutrophil gelatinaseassociated lipocalin (NGAL), tumor necrosis factor-like inducer of apoptosis (TWEAK), in addition to other better known biomarkers including anti-C1q antibody, antidsDNA antibody, Antinucleosome Antibody, C3 and C4. However, no widely-accepted definition of the disease state and clinical outcome measures is proposed by these studies [14].

The treatment of severe LN, usually class III, IV and V, consists two phases. Intensive immunosuppressive medication is used in the first phase (induction phase) to achieve early remission. Less intensive immunosuppressive regimen is involved in the second phase (maintenance phase) to prevent further deterioration of renal function.

For patients with class III or IV LN, glucocorticosteroids is recommended in combination with either cyclophosphamide or mycophenolate mofetil for the induction phase. High dose of glucocorticosteroids is adopted during the induction phase, however, some evidence suggests that lower initial dose of corticosteroids may be enough to induce early remission $[15,16]$. Given the less severe side effects, mycophenolate mofetil is preferred for induction phase. The efficacy of mycophenolate mofetil to induce remission is similar to that of cyclophosphamide [17]. However, cyclophosphamide is favorable in case of severe deterioration of renal function [12]. During the maintenance phase, low dose of steroids and mycophenolate mofetil are the first options.

The patient in this case report was given high dose of methylprednison and cyclophosphamide for her induction phase, in conjunction with urgent hemodialysis. In addition, lisinopril was added with the target of severe proteinuria.

\section{CONCLUSION}

In summary, $\mathrm{LN}$ is unlikely when a combination of anti-C1q antibody, anti-ds DNA antibody and C3. C4 level is within normal range, considering the good negative predictive value. However, the definite diagnosis of LN is made by kidney biopsy with optical microscope, immunofluorescence techniques and an electron microscope analysis. It is not unusual to have patients with normal immunologic profile but active LN on biopsy studies, which require the aggressive treatment to prevent the further deterioration of the renal function.

\section{REFERENCES}

[1] Mok, C.C. (2012) Understanding lupus nephritis: Diag- 
nosis, management, and treatment options. International Journal of Women's Health, 4, 213-222.

[2] Mok, C.C. (2006) Therapeutic options for resistant lupus nephritis. Seminars in Arthritis and Rheumatism, 36, 7181. http://dx.doi.org/10.1016/j.semarthrit.2006.04.008

[3] Bertsias, G., Gordon, C. and Boumpas, D.T. (2008) Clinical trials in systemic lupus erythematosus (SLE): Lessons from the past as we proceed to the future-The EULAR recommendations for the management of SLE and the use of end-points in clinical trials. Lupus, 17, 437-442. http://dx.doi.org/10.1177/0961203308090031

[4] Weening, J.J., D’Agati, V.D., Schwartz, M.M., Seshan, S.V., Alpers, C.E., Appel, G.B., Balow, J.E., Bruijn, J.A., Cook, T., Ferrario, F., Fogo, A.B., Ginzler, E.M., Hebert, L., Hill, G., Hill, P., Jennette, J.C., Kong, N.C., Lesavre, P., Lockshin, M., Looi, L.M., Makino, H., Moura, L.A. and Nagata, M. (2004) The classification of glomerulonephritis in systemic lupus erythematosus revisited. Journal of the American Society of Nephrology, 15, 241- 250. http://dx.doi.org/10.1097/01.ASN.0000108969.21691.5D

[5] Mittal, B., Rennke, H. and Singh, A.K. (2005) The role of kidney biopsy in the management of lupus nephritis. Current Opinion in Nephrology and Hypertension, 14, 18. http://dx.doi.org/10.1097/00041552-200501000-00002

[6] Sinico, R.A., Radice, A., Ikehata, M., Giammarresi, G., Corace, C., Arrigo, G., Bollini, B. and Li, V.M. (2005) Anti-C1q autoantibodies in lupus nephritis: Prevalence and clinical significance. Annals of the New York Academy of Sciences, 1050, 193-200.

http://dx.doi.org/10.1196/annals.1313.020

[7] Moroni, G., Radice, A., Giammarresi, G., Quaglini, S., Gallelli, B., Leoni, A., Li Vecchi, M., Messa, P. and Sinico, R.A. (2009) Are laboratory tests useful for monitoring the activity of lupus nephritis? A 6-year prospective study in a cohort of 228 patients with lupus nephritis. Annals of the Rheumatic Diseases, 68, 234-237. http://dx.doi.org/10.1136/ard.2008.094508

[8] Swaak, A.J., Groenwold, J. and Bronsveld, W. (1986) Predictive value of complement profiles and anti-dsDNA in systemic lupus erythematosus. Annals of the Rheumatic Diseases, 45, 359-366. http://dx.doi.org/10.1136/ard.45.5.359

[9] Christopher-Stine, L., Siedner, M., Lin, J., Haas, M., Parekh, H., Petri, M. and Fine, D.M. (2007) Renal biopsy in lupus patients with low levels of proteinuria. The Journal of Rheumatology, 34, 332-335.

[10] Cameron, J.S. (1999) Lupus nephritis. Journal of the American Society of Nephrology, 10, 413-424.

[11] Contreras, G., Pardo, V., Cely, C., Borja, E., Hurtado, A., De La Cuesta, C., Iqbal, K., Lenz, O., Asif, A., Nahar, N.,
Leclerq, B., Leon, C., Schulman, I., Ramirez-Seijas, F., Paredes, A., Cepero, A., Khan, T., Pachon, F., Tozman, E., Barreto, G., Hoffman, D., Almeida Suarez, M., Busse, J.C., Esquenazi, M., Esquenazi, A., Garcia Mayol, L. and Garcia, E.H. (2005) Factors associated with poor outcomes in patients with lupus nephritis. Lupus, 14, 890895. http://dx.doi.org/10.1191/0961203305lu2238oa

[12] Ruiz Irastorza, G., Espinosa, G., Frutos, M.A., Jimenez Alonso, J., Praga, M., Pallares, L., Rivera, F., Robles Marhuenda, A., Segarra, A. and Quereda, C. (2012) Diagnosis and treatment of lupus nephritis. Consensus document from the systemic auto-immune disease group (GEAS) of the Spanish Society of Internal Medicine (SEMI) and Spanish Society of Nephrology (S.E.N.). Nefrologia, 32, 1-35.

[13] Mok, C.C. (2010) Biomarkers for lupus nephritis: A critical appraisal. Journal of Biomedicine and Biotechnology, 2010, Article ID: 638413. http://dx.doi.org/10.1155/2010/638413

[14] Davidson, A. and Aranow, C. (2006) Pathogenesis and treatment of systemic lupus erythematosus nephritis. Current Opinion in Rheumatology, 18, 468-475.

[15] Houssiau, F.A., Vasconcelos, C., D’Cruz, D., Sebastiani, G.D., Garrido Ed Ede, R., Danieli, M.G., Abramovicz, D., Blockmans, D., Mathieu, A., Direskeneli, H., Galeazzi, M., Gul, A., Levy, Y., Petera, P., Popovic, R., Petrovic, R., Sinico, R.A., Cattaneo, R., Font, J., Depresseux, G., Cosyns, J.P. and Cervera, R. (2002) Immunosuppressive therapy in lupus nephritis: The Euro-Lupus Nephritis Trial, a randomized trial of low-dose versus high-dose intravenous cyclophosphamide. Arthritis \& Rheumatism, 46, 2121-2131. http://dx.doi.org/10.1002/art.10461

[16] Illei, G.G., Austin, H.A., Crane, M., Collins, L., Gourley, M.F., Yarboro, C.H., Vaughan, E.M., Kuroiwa, T., Danning, C.L., Steinberg, A.D., Klippel, J.H., Balow, J.E. and Boumpas, D.T. (2001) Combination therapy with pulse cyclophosphamide plus pulse methylprednisolone improves long-term renal outcome without adding toxicity in patients with lupus nephritis. Annals of Internal Medicine, 135, 248-257.

http://dx.doi.org/10.7326/0003-4819-135-4-200108210-0 $\underline{0009}$

[17] Appel, G.B., Contreras, G., Dooley, M.A., Ginzler, E.M., Isenberg, D., Jayne, D., Li, L.S., Mysler, E., SanchezGuerrero, J., Solomons, N. and Wofsy, D. (2009) Mycophenolate mofetil versus cyclophosphamide for induction treatment of lupus nephritis. Journal of the American Society of Nephrology, 20, 1103-1112. http://dx.doi.org/10.1681/ASN.2008101028 